\title{
A gut-secreted peptide controls arousability through modulation of dopaminergic neurons in the brain
}

\author{
Authors: Iris Titos ${ }^{1}$, Dragana Rogulja ${ }^{1 *}$ \\ ${ }^{1}$ Department of Neurobiology, Harvard Medical School, Boston, MA 02115. *Correspondence \\ to: Dragana_Rogulja@hms.harvard.edu
}


Since sensory information is always present in the environment, animals need to internally regulate their responsiveness to fit the context. During sleep, the threshold for sensory arousal is increased so that only stimuli of sufficient magnitude can cross it. The mechanisms that make arousability flexible are largely mysterious, but they must integrate sensory information with information about physiology. We discovered a gut-to-brain signaling pathway that uses information about ingested nutrients to control arousability from sleep, without affecting sleep duration. Protein ingestion causes endocrine cells in the Drosophila gut to increase production of $\mathrm{CCHa} 1$, a peptide that decreases sensory responsiveness. $\mathrm{CCHa} 1$ is received by a small group of brain dopaminergic neurons whose activity gates behavioral responsiveness to mechanical stimulation. These dopaminergic neurons innervate the mushroom body, a brain structure involved in determining sleep duration. This work describes how the gut tunes arousability according to nutrient availability, allowing deeper sleep when dietary proteins are abundant. It also suggests that behavioral flexibility is increased through independent tuning of sleep depth

15 and duration.

Arousability is dynamically regulated, allowing context-appropriate behavioral responsiveness and facilitating deep sleep. Flies share with mammals all the behavioral hallmarks of sleep, including decreased responsiveness to stimuli ${ }^{1,2}$. While work in flies led to identification of neural circuits and conserved genes that regulate sleep duration ${ }^{3}$, much less is known about the regulation of sleep depth ${ }^{4-8}$. Since the restorative function of sleep depends on its quality and not just duration ${ }^{9}$, it is important to identify the molecular and circuit mechanisms that set arousal

23 threshold.

We probed the arousal threshold of sleeping flies with mechanical stimulation. In our setup, loudspeakers convert a tunable electrical signal into 2-second-long vibrations every 30-40 minutes throughout the night (intervals are randomized to minimize habituation). Responsiveness

28 to vibrations is automatically tracked for individual animals (Extended Data Fig. 1a,b, and 29 Experimental Procedures). We determined the amplitudes sufficient to wake up either $\sim 20 \%$ or $30 \sim 95 \%$ of control flies and used these settings to screen for genetic manipulations that increased 31 or decreased sensory responsiveness. We used elav-Gal4, a driver mainly expressed in 32 neurons ${ }^{10}$, to target $\sim 3,400$ genes with RNAi (the screen was unbiased). The screen produced 33 both hyper- and hypo-arousable phenotypes (Fig. 1a) (Extended Data Table 1). A Gene 34 Ontology (GO) term analysis (see Experimental Procedures) revealed diversity in biological 35 processes, molecular functions and cellular localization for the genes whose mRNA knockdown 
36 produced arousability phenotypes (Extended Data Fig.1c). We focused on the neuropeptide

37 CCHamide-1 and its receptor $\mathrm{CCHa} 1 \mathrm{R}$, since both have strong phenotypes and belong to the

38 same pathway. $\mathrm{CCHamides}(\mathrm{CCHa} 1$ and $\mathrm{CCHa} 2)$ are recently discovered neuropeptides with

39 functions related to feeding and growth regulation ( $\mathrm{CCHa} 2)$, and olfactory responsiveness and

40 circadian behavior $(\mathrm{CCHa} 1)^{11-16}$. $\mathrm{CCHa} 1 \mathrm{R}$ is a $\mathrm{G}$ protein-coupled receptor homologous to the

41 mammalian Gastrin-Releasing Peptide Receptor ${ }^{16}$ which regulates itch sensation ${ }^{17}$ and

42 responsiveness to social interactions ${ }^{18,19}$.

44 RNAi against either $\mathrm{CCHa} 1$ or $\mathrm{CCHa} 1 \mathrm{R}$ led to hyper-arousability, with $\sim 85 \%$ of animals waking 45 up in response to low-amplitude stimulation that woke up only $\sim 20 \%$ of the parental controls (Fig.

46 1b, elav>CCHa1-RNAi and elav>CCHa1R-RNAi). We noticed that either the peptide or the 47 receptor depletion caused flies to respond more readily to stimulation even when awake (as 48 reported by locomotor response upon stimulation), without increasing basal locomotion

49 (Extended Data Fig. 2a). If undisturbed, CCHa1-R- knockdown animals slept less than the 50 controls and CCHa1 knockdown flies had more fragmented sleep (Extended Data Fig. 2a). The 51 specificity of RNAi for $\mathrm{CCHa} 1$ and $\mathrm{CCHa} 1 \mathrm{R}$ (and not $\mathrm{CCHa} 2$ and $\mathrm{CCHa} 2 \mathrm{R}$ ) was shown with 52 quantitative RT-PCR (Extended Data Fig. 2b). Additional RNAi lines (Extended Data Fig 2c,d), 53 as well as transgenic flies containing the MiMIC constructs from the Gene Disruption Project 54 (Mi\{MIC\}CCHa1 ${ }^{\mathrm{M} 109190}$ and Mi\{MIC\}CCHa1R ${ }^{\mathrm{MI08118}}$ that affect $\mathrm{CCHa} 1$ and $\mathrm{CCHa} 1 \mathrm{R}^{20}$ respectively)

55 (Fig. 1C and Extended Data Fig. 2e), confirmed the role for these genes in arousability. Total 56 sleep was somewhat reduced. A conditional approach allowed depletion of $\mathrm{CCHa} 1$ and $\mathrm{CCHa1R}$ 57 during adulthood, which was sufficient to cause hyper-arousability (Extended Data Fig. 2f). We 58 concluded that $\mathrm{CCHa}$, likely signaling through $\mathrm{CCHa1R}$, suppresses sensory arousability in 59 adults (Fig. 1d).

61 To find the relevant $\mathrm{CCHa}$-producing cells, we raised antibodies against CCHa1 (using a 62 previously validated epitope ${ }^{21}$ ) and expressed $\mathrm{CCHa1-RNAi} \mathrm{with} \mathrm{spatially} \mathrm{restricted} \mathrm{Gal4s.} \mathrm{The}$ 63 antibody signal agreed with previous reports ${ }^{13,16,21}$ (Fig. 2a) and with the pattern of CCHa164 promoter-driven GFP (Extended Data Fig. 3a). The signal was abolished in elav>CCHa1-RNAi 65 flies (Fig. 2a) and in homozygous CCHa1 mutants (Extended Data Fig. 3a). From 100 Gal4 66 lines, we found one that recapitulated the phenotype obtained with elav-Gal4 (Fig. 2b). This line 67 did not noticeably change, or even overlap with, the $\mathrm{CCHa} 1$ signal in the nervous system 68 (EECG>CCHa1-RNAi in Fig. 2a, EECG>GFP in Extended Data Fig. 3b), indicating that the 69 hyper-arousability phenotype seen with $\mathrm{CCHa} 1$ depletion may have originated in a non-neuronal 
70 tissue. $\mathrm{CCHa} 1$ was previously detected in a posterior subpopulation of enteroendocrine cells ${ }^{21}$ a

71 type of secretory cell sparsely distributed throughout the gut ${ }^{22}$ (Extended Data Fig. 3c).

72 Enteroendocrine cells and neurons share developmental programs ${ }^{22}$, which could explain why

73 elav-Gal4, the pan-neuronal driver we used for RNAi expression, is also expressed in

74 enteroendocrine cells $^{23}$ (Extended Data Fig. 3d). The $\mathrm{CCHa} 1$ signal in the gut indeed

75 disappeared when CCHa1-RNAi was driven by elav-Gal4 (Fig. 2a and Extended Data Fig. 3e).

76 The restricted driver that produced the arousal phenotype without altering neuronal CCHa1 levels

77 also depleted $\mathrm{CCHa} 1$ from the gut (Fig. 2a,b and Extended Data Fig. 3e). We call this driver

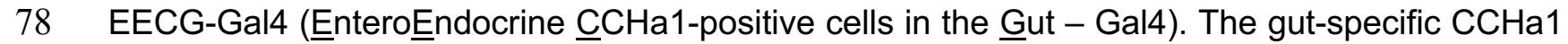

79 depletion (EECG>CCHa1-RNAi) led to increased arousability both during sleep and wakefulness

80 (Fig. 2b and Extended Data Fig. 3f), similarly to what was seen with elav-Gal4 (Fig. 1b and

81 Extended Data Fig. 2a). All the phenotypes observed with EECG-Gal4 were reproduced with a

82 conditional version of pros-Gal4 $4^{24}$, (Fig. 2a,b and Extended Data Fig. 3b,e and g) a classic driver

83 for enteroendocrine cells. Depletion of $\mathrm{CCHa} 1$ from the gut had no effect on daily sleep amount,

84 or on how much food animals ingested (Extended Data Fig. 3f, $\mathbf{g}$ and $\mathbf{h}$ ). As there was no change

85 in sleep duration, we could study specifically how gut-produced $\mathrm{CCHa} 1$ regulates arousability (i.e.

86 sleep depth).

Mammalian enteroendocrine cells are electrically excitable ${ }^{25}$. In Drosophila, activation of enteroendocrine cells increases intracellular calcium and promotes vesicle release ${ }^{26}$. We used neuronal activation and silencing tools to ask whether changing the activity of enteroendocrine cells can also impact arousability. Conditional silencing (with a temperature-sensitive allele of a

92 dynamin homolog shibire, shits27) or conditional activation (with a temperature-sensitive cation

93 channel $\operatorname{TrpA}^{28}$ ) produced strong and opposite phenotypes (Fig. 2c,d). Silencing EECGs

94 phenocopied $\mathrm{CCHa} 1$ depletion, with animals reacting more readily to vibrations (Fig. 2c and

95 Extended Data Fig. 4a). When enteroendocrine cells were activated (using EECG-Gal4 or pros-

96 Gal4;Tub-Gal80 ${ }^{\text {ts }}$ ) animals were harder to arouse with vibrations (Fig. 2d). The phenotype was

97 suppressed if the cells could not produce $\mathrm{CCHa} 1$, demonstrating that $\mathrm{CCHa} 1$ mediates the effect

98 of enteroendocrine cells on arousability (Fig. 2d and Extended Data Fig. 4b and 5). Activation

99 of enteroendocrine cells reduced total sleep in a CCHa1-independent manner (Extended Data

100 Fig. 4b and 5) suggesting that non-CCHa1 cells labeled with those Gal4s could have a role in 101 regulating sleep duration. We concluded that enteroendocrine cells use the peptide $\mathrm{CCHa} 1$ to 102 control arousability (Fig. 2e). 
104 Gut cells are positioned to absorb nutrients and assess food quality, and peptides produced and

105 secreted by the enteroendocrine cells could be used to propagate this information ${ }^{14,22,29}$. Food

106 satiety may increase not only sleep duration ${ }^{30-32}$ but also sleep depth by suppressing arousability.

107 We asked if the activity of enteroendocrine cells, and their production of $\mathrm{CCHa} 1$, changes in

108 response to three basic dietary components: sugar, fat and protein. To measure cell activity, we

109 used pros-Gal4 to drive CaLexA ${ }^{33}$ (Fig. 3a), a GFP-based reporter previously shown to register

110 calcium changes in the fat body, neurons and enteroendocrine cells ${ }^{26}$. Supplementing standard

111 fly food with sugars (glucose, galactose or fructose) or fat (coconut oil, propionic acid or hexanoic

112 acid) had no effect at the tested concentrations; in contrast, with peptone as an additional protein

113 source, the activity of $\mathrm{CCHa} 1$-positive enteroendocrine cells was increased, as were their $\mathrm{CCHa} 1$

114 levels (Fig. 3b,c and Extended Data Fig. 6a). The magnitude of the response depended on how

115 long the flies had access to supplemented food, since the increase in CCHa1 levels was stronger

116 after 24 hours than after 6 hours of peptone feeding (Extended Data Fig. 6b). Not all cells with

117 high $\mathrm{CCHa} 1$ levels showed evidence of activation, and some activated cells did not label for

118 CCHa1 (Fig. 3b); the latter observation suggests that other peptides made in this area of the gut

119 could signal protein ingestion and affect behavior.

121 Peptones are made by partial hydrolysis of dietary proteins and contain polypeptides and amino

122 acids. To investigate if specific peptone components activate $\mathrm{CCHa} 1$-producing enteroendocrine

123 cells, we supplemented regular food with different combinations of amino acids, matching their

124 respective concentrations in peptone. Adding single essential vs non-essential or biochemically-

125 related amino acids to the food did not recapitulate the $\mathrm{CCHa} 1$ increase observed with peptone

126 (Extended Data Fig. 6c). However, the mix of all amino acids (which matched the total amino

127 acid concentration without changing their individual representation with respect to peptone)

128 strongly induced CCHa1 production (Extended Data Fig. 6c). The most likely explanation is that

129 EECGs do not receive signals from specific amino acids but monitor whether food is generally

130 protein-rich (Extended Data Fig. 6d), and adjust arousability accordingly. Peptone

131 supplementation indeed suppressed sensory arousal; only half as many wild-type flies fed

132 peptone-supplemented food woke up in response to vibrations compared to flies fed regular food

133 (Fig. 3d). Peptone feeding had a modest effect on responsiveness during wakefulness, basal

134 locomotion, sleep duration and feeding (Extended Data Fig. 6e and f). To determine whether

$135 \mathrm{CCHa} 1$ mediates the effect of peptone on arousal, we repeated the feeding experiments in

136 animals lacking $\mathrm{CCHa} 1$ in the gut. Because removal of $\mathrm{CCHa} 1$ by itself causes hyper-arousability,

137 lower amplitude stimulation was used in animals lacking the peptide (EECG>CCHa1-RNAi and 
138 pros-Gal4:TubGal80 ${ }^{\text {ts }}>\mathrm{CCHa} 1-\mathrm{RNAi}$ ) than in the parental controls. This way, baseline arousability 139 (on regular food) could be matched across genotypes (Fig. 3e and Extended Data Fig. 6g). $140 \mathrm{CCHa1}$ knockdown weakened the effect of peptone on arousal (Fig. 3e and Extended Data Fig.

$141 \mathbf{6 g}$ ), though incomplete phenotype suppression reinforces the idea that dietary proteins activate 142 multiple signaling pathways. Together, our results show that ingested proteins activate $\mathrm{CCHa1-}$ 143 producing cells in the gut to suppress arousability (Fig. 3f).

145 Peptides produced by enteroendocrine cells can either signal through synapses or be secreted 146 into circulation ${ }^{22}$. CCHa1-producing cells reside in a poorly innervated region ${ }^{22}$, suggesting that $147 \mathrm{CCHa} 1$ works as a hormone. We looked for $\mathrm{CCH} 1$-receiving cells by knocking down the receptor 148 with different Gal4 drivers and testing arousability. Flies were not hyper-arousable when CCHa1R 149 was knocked down in cholinergic (Cha-Gal4), octopaminergic (Tdc2-Gal4), glutamatergic (vGlut150 Gal4), or circadian (Tim-Gal4) neurons (Extended Data Fig. 7a). Though one dopaminergic 151 driver (TH-Gal4) produced no phenotype, another dopaminergic driver with a broader expression 152 pattern (Ddc-Gal4) produced a strong phenotype (Fig. 4a). TH-Gal4 covers the majority of 153 dopamine neurons but spares the PAM cluster, labeled by Ddc-Gal4 ${ }^{34}$. Flies were indeed hyper154 arousable when CCHa1R-RNAi was driven in the $\sim 87$ neurons $^{34}$ that comprise the PAM 155 dopaminergic cluster in each brain hemisphere (PAM-Gal4) (Fig. 4a). Arousability during 156 wakefulness was weakly increased, while basal locomotion and sleep duration were unaffected 157 by these manipulations (Extended Data Fig. 7b). Two different Gal4s expressed under the 158 control of $\mathrm{CCHa1R}$ regulatory elements label the PAM dopaminergic cluster (Extended Data Fig. 159 7c), agreeing with the notion that the receptor is expressed there.

161 Thanks to a large-scale mapping and tool-generating effort ${ }^{35}$, access to subpopulations of PAM 162 neurons is possible, which allowed us to further narrow down the CCHa1 target cells. Subsets of 163 PAM neurons have been implicated in regulating sleep duration and food foraging ${ }^{36-38}$. We found 164 that depleting $\mathrm{CCHa1R}$ from a small PAM subpopulation labeled by PAM ${ }^{\mathrm{MB} 441 \mathrm{~B}}-\mathrm{Gal}^{35}{ }^{35}$ caused 165 flies to wake up more easily (Fig. 4b). PAM ${ }^{\text {MB441B }}$-Gal4 labels $~ 8-10$ neurons per hemisphere and 166 has no expression in the gut (Fig. 4b and Extended Data Fig. 7d). Recently, those neurons were 167 found to express $\mathrm{CCHa} 1 \mathrm{R}^{39}$. Depletion of $\mathrm{CCHa} 1 \mathrm{R}$ from $\mathrm{PAM}^{\mathrm{MB} 441 \mathrm{~B}}$ neurons did not change sleep 168 duration or feeding, but it decreased basal locomotion (Extended Data Fig. 7e,f). In contrast to 169 all other manipulations described so far, depleting $\mathrm{CCHa1R}$ from this narrow dopaminergic cell 170 population did not affect responsiveness of awake flies (Extended Data Fig. 7e). This suggests 171 that $\mathrm{CCHa} 1$ is received by a separate population of $\mathrm{CCHa1R}$-expressing dopaminergic neurons 
172 in the PAM cluster to regulate arousability during wakefulness, meaning that sensory gating

173 during sleep and wakefulness can be uncoupled.

174

175 If brain dopaminergic neurons receive $\mathrm{CCHa} 1$ from the gut, their activity should change in 176 response to inputs that activate the $\mathrm{CCHa1-producing} \mathrm{enteroendocrine} \mathrm{cells,} \mathrm{in} \mathrm{a} \mathrm{CCHa1R}$ 177 dependent manner; further, behavioral effects stemming from increased CCHa1 production 178 should be abolished by $\mathrm{PAM}^{\mathrm{MB} 441 \mathrm{~B}}$-specific $\mathrm{CCHa1R}$ depletion. In agreement, peptone 179 supplementation nearly doubled the calcium-activated GFP signal in PAM ${ }^{\text {MB441B }}$ neurons (Fig. 180 4c), and this was dependent on $\mathrm{CCHa1R} \mathrm{PAM}^{\mathrm{MB} 441 \mathrm{~B}}$-specific $\mathrm{CCHa1R}$ depletion prevented the 181 neurons from being activated by dietary peptone (Fig. 4c). This manipulation also abolished the 182 effect of peptone feeding on arousability (which was specific for sleep state) (Fig. 4d and 183 Extended Data Fig. 7g). Next, we investigated how the activity of PAM ${ }^{\text {MB } 441 B}$ neurons affects sensory responsiveness. Conditional silencing of these neurons caused hyper-arousability, while their conditional activation caused hypo-arousability, specifically during sleep (Fig. $\mathbf{4 e}$ and

186 Extended Data Fig. 8a). Sleep duration and locomotion were unaffected (Extended Data Fig.

187 8a). Knocking down Tyrosine Hydroxylase ( $\mathrm{TH}$, the rate-limiting enzyme in dopamine synthesis) 188 in PAM $^{\mathrm{MB} 441 \mathrm{~B}}$ neurons caused hyper-arousability during sleep (Fig. 4f), arguing that dopamine 189 mediates the effect of $\mathrm{PAM}^{\mathrm{MB} 441 \mathrm{~B}}$ neurons on arousal. Again, sleep duration and responsiveness 190 during wakefulness were unchanged (Extended Data Fig. 8b). Dopamine, perhaps the best 191 known arousal-promoting molecule ${ }^{40-43}$ can therefore suppress arousal by acting on specific 192 synapses.

194 The PAM dopaminergic neurons, including the MB441B population, project to the mushroom 195 body, a brain structure that receives sensory inputs from different modalities ${ }^{44-49}$ and regulates 196 olfaction, memory and sleep duration ${ }^{38,50-55}$. PAM neurons modulate synapses between Kenyon 197 cells (mushroom body intrinsic neurons) and the mushroom body output neurons (MBONs) ${ }^{56}$ to 198 contextualize and drive appropriate behavioral responses ${ }^{38,56}$. So far, we talked about a 199 physiological signal (CCHa1) acting on modulatory neurons (dopaminergic PAMs). But where 200 does the sensory information itself fit in, where is it received in the system? Kenyon cells carry 201 olfactory and visual information ${ }^{49,57}$ but there is no evidence yet that they receive mechanical 202 inputs. The larval ${ }^{58}$ and adult ${ }^{59}$ PAM neurons on the other hand are known to receive such 203 information. We targeted CaLexA to $\mathrm{PAM}^{\mathrm{MB} 441 \mathrm{~B}}$ neurons and exposed flies to mechanical stimuli 204 used to test arousal threshold (Fig. 4g). The GFP signal in the PAM ${ }^{\text {MB441B }}$ cell bodies was 205 quantified 4 hours after stimulation (which should be enough time for detecting an increase in 
206 GFP signal), in case neurons are activated, as well as 28 hours after stimulation (which should 207 be enough time for GFP degradation and signal decrease, in case neuronal activity is reduced ${ }^{60}$ ). 208 CaLexA reported that $\mathrm{PAM}^{\mathrm{MB} 441 \mathrm{~B}}$ neurons are inhibited in response to vibrations (Fig. $\mathbf{4 g}$ ). These 209 results suggest that $\mathrm{PAM}^{\mathrm{MB} 441 \mathrm{~B}}$ neurons could regulate arousal by integrating mechanosensory 210 information with a physiological signal from the gut. Arousability is attenuated across sensory 211 modalities during sleep ${ }^{33}$; an outstanding question is whether different senses can be tuned 212 independently. To address this, we asked whether the CCHa1 pathway regulates arousability in 213 response to other sensory stimuli besides mechanical vibrations. We built a setup for rapid 214 temperature increase that was effective at waking flies up with heat $\left(35-40{ }^{\circ} \mathrm{C}\right)$ (Extended Data

215 Fig. 9a). Unlike for vibrations, the $\mathrm{CCHa} 1$ pathway had no bearing on the ability of thermal stimuli 216 to elicit arousal (Extended Data Fig. 9b). In agreement with behavioral data, there was no change 217 in the activity of PAM ${ }^{\mathrm{MB} 441 \mathrm{~B}}$ neurons when flies were exposed to thermal stimuli (Extended Data 218 Fig. 9c). Gating of different sensory modalities is therefore modular, perhaps allowing greater 219 flexibility in adjusting behaviors to external and internal circumstances.

$221 \mathrm{PAM}^{\mathrm{MB} 441 \mathrm{~B}}$ neurons project to the $\mathrm{Y} 3$ compartment of the mushroom body ${ }^{35}$. In each brain

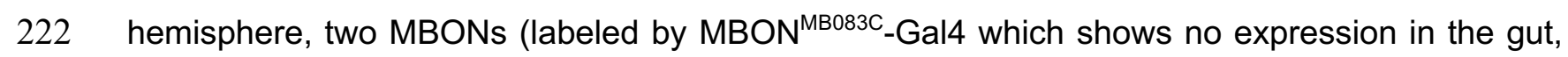
223 (Fig. 4h and Extended Data Fig. 9d)) receive information from this compartment and transmit it 224 to other brain centers ${ }^{35,38}$. Conditional silencing of the MBON ${ }^{\text {MB083C }}$ caused flies to be easily 225 awoken with vibrations, while conditional activation had the opposite effect (Fig. 4h). 226 Responsiveness was not changed in awake animals (Extended Data Fig. 9e). In contrast to the 227 upstream $\mathrm{CCHa1}$ pathway components we described, $\mathrm{MBON}^{\mathrm{MB} 083 \mathrm{C}}$ also have a role in 228 determining sleep duration: changing their activity can bidirectionally modulate the amount of 229 sleep (Extended Data Fig. 9e). Other studies have reported that altering the activity of Kenyon 230 cells or MBON can impact sleep duration ${ }^{3}$. Our results point to convergence of circuits that control 231 sleep amount and sleep depth. They also specifically demonstrate that dietary proteins boost 232 production of $\mathrm{CCHa} 1$ in the gut, and that $\mathrm{CCHa} 1$ targets dopaminergic neurons in the brain to 233 suppress arousability (Fig. 4i).

Animals need to decide which of the many environmental cues are worth responding to. Elevated 236 arousal threshold is a core feature of sleep ${ }^{61}$ and the mechanisms that allow flexible arousability 237 are likely conserved. In mammals, the flow of sensory information is thought to be gated by the 238 thalamus ${ }^{57,62}$ but olfactory information can be modulated despite not passing through this brain 239 region $^{63}$. This suggests that different sensory modalities can be regulated independently, a view 
240 supported by our results. Multiple sensory streams are received by the mushroom body ${ }^{35,49}$. This 241 brain structure is organized in a modular way ${ }^{35}$, which might be particularly suited for modular 242 processing of sensory cues. Dopamine itself is a key regulator of arousal across species ${ }^{64}$ but $^{2}$ 243 there is little understanding of how the dopaminergic system itself is regulated. We describe a 244 pathway that informs specific dopaminergic neurons, which seem to also receive sensory 245 information, about ingested nutrients, thereby tuning them to physiology (Fig. $\mathbf{4 j}$ ). The logic of the 246 system has similarities with the dopaminergic tuning of sexual arousal by signals from the 247 reproductive periphery ${ }^{65,66}$. Abundance of molecules from food could signal an opportunity to 248 sleep more deeply since there is no need to forage; peptides like $\mathrm{CCHa} 1$ generally have longer249 lasting effects than classical neurotransmitters ${ }^{67}$ and are ideal for regulating states like sleep that 250 can last for hours ${ }^{3}$. Nutritional status is a critical variable for many behavioral outcomes so there 251 are likely to be different signaling axes like the one we describe, affecting multiple behaviors. The 252 molecular and functional diversity of gut endocrine cells is increasingly appreciated as 253 fundamental for regulating processes other than digestion ${ }^{22}$. The gut influences behavior more 254 profoundly than previously appreciated through peptide signals that can diffuse to the brain or 255 through direct connections with the nervous system (notably via the vagus nerve ${ }^{68}$ ). Our study 256 adds to the emerging knowledge on the basic mechanisms of gut-brain interactions ${ }^{22}$. Recent 257 evidence links gut dysfunction with neurological diseases like the autism spectrum disorders, 258 Parkinson's and Alzheimer's disease ${ }^{69}$. Together, these studies support the centuries-old idea 259 that digestive and nervous systems are critically linked in normal physiology and disease ${ }^{70}$. 


\section{ACKNOWLEDGEMENTS}

263 We thank our lab and Michael Crickmore's lab for advice and comments on the manuscript. Ofer

264 Mazor and Pavel Gorelik assisted in building the arousal threshold setup. Benjamin Sanchez

265 developed the software used to control mechanical stimuli and analyze the behavioral

266 responsiveness. Keishi Nambara and Alexa Soares assisted with the screen. For fly stocks, we

267 thank Michael Crickmore, Nicholas Stavropoulos, Kyunghee Koh and Norbert Perrimon. We thank

268 Pedro Saavedra for assistance with the quantitative RT-PCR. I.T. was supported by the Mahoney

269 and Brooks postdoctoral fellowships. D.R. is a New York Stem Cell - Robertson investigator. This

270 work was supported by the New York Stem Cell Foundation, the NIH (DP2 OD022385) and the

271 Pew Scholars Program in the Biomedical Sciences.

\section{AUTHOR CONTRIBUTIONS}

274 I.T. and D.R. designed the study. I.T. performed the experiments. I.T. and D.R. analyzed the data

275 and wrote the paper.

\section{DECLARATION OF INTERESTS}

278 The authors declare no conflict of interest.

\section{FIGURES AND FIGURE LEGENDS}

Figure 1. CCHa1 and CCHa1R suppress arousability. (a) Results of a screen for genes that regulate arousability. Low amplitude stimulus uncovers hyper-responsive phenotypes, while high amplitude stimulus uncovers hypo-responsive phenotypes (phenotypes exceed two standard deviations from the mean). (b) elav-Gal4-driven RNAi against $\mathrm{CCHa}$ (elav>CCHa1-RNAi) or its receptor (elav>CCHa1R-RNAi) increases arousability of sleeping animals. (c) $\mathrm{CCHa} 1$ and CCHa1R mutant flies are easier to wake up during sleep than control flies. (d) Schematic: CCHa1 and CCHa1R suppress arousability. In all panels, mean and S.E.M. are depicted; sample sizes and statistical analyses are shown in Extended Data Table 2.

$301 \mu \mathrm{m}$. 
Figure 3. $\mathrm{CCHa} 1$ in enteroendocrine cells responds to dietary proteins. (a) The CaLexA method detects calcium-dependent cell activation. (b) CCHa1 mRNA (measured by qRT-PCR, each dot represents 10 guts) and protein levels, and CaLexA-dependent GFP signal, increase in the posterior midgut when flies are fed protein-enriched food. (c) Supplementing regular food with protein (peptone), but not fat (coconut oil) or sugar (glucose), activates enteroendocrine cells and increases $\mathrm{CCHa} 1$ protein and mRNA levels. (d) Protein-enriched diet decreases arousability. (e) Knocking down CCHa1 specifically in the gut with EECG-Gal4 or pros-Gal4:Tub-Gal80 ${ }^{\text {ts }}$ diminishes the peptone-induced changes in arousability. (f) Schematic: protein ingestion activates $\mathrm{CCHa} 1$-producing cells and increases the neuropeptide levels to suppress arousability. In all panels, mean and S.E.M. are depicted; sample sizes and statistical analyses are in Extended Data Table 2. Scale bars: $100 \mu \mathrm{m}$.

Figure 4. $\mathrm{CCHa} 1$ regulates arousability through dopaminergic neurons in the brain. (a) Knockdown of the $\mathrm{CCHa} 1$ receptor using dopaminergic drivers increases arousability from sleep. (b) $\mathrm{PAM}^{\mathrm{MB} 441 \mathrm{~B}}-\mathrm{Gal} 4$ expression in the brain visualized with a membrane-targeted GFP. Presynaptic protein Brp marks the neuropil. Dashed white line marks the mushroom bodies. Schematic of the expression pattern of the Gal4s used in this figure. Knocking down CCHa1 receptor in $\mathrm{PAM}^{\mathrm{MB} 441 \mathrm{~B}}$ neurons increases arousability from sleep. (c) Protein-enriched diet activates PAM ${ }^{\mathrm{MB} 441 \mathrm{~B}}$ neurons, as visualized with a calcium-dependent GFP reporter CaLexA, in a CCHa1R-dependent manner. (d) Knocking down $\mathrm{CCHa} 1 \mathrm{R}$ in $\mathrm{PAM}^{\mathrm{MB} 441 \mathrm{~B}}$ neurons diminishes the peptone-induced changes in arousability. (e) Inhibition (with shits) and activation (with TrpA1) of $\mathrm{PAM}^{\mathrm{MB} 441 \mathrm{~B}}$ neurons increases and decreases arousability, respectively. (f) Knocking down tyrosine hydroxylase (TH) in PAM ${ }^{\mathrm{MB} 441 \mathrm{~B}}$ neurons increases arousability from sleep. (g) Mechanical vibrations reduce the activity of PAM ${ }^{\mathrm{MB} 441 \mathrm{~B}}$ neurons, as visualized with a calcium-dependent GFP reporter CaLexA 4 and 28 hours after exposure to mechanosensory stimuli. (h) Mushroom body output neurons from the $\mathrm{\gamma} 3$ compartment, $\mathrm{MBON}^{\mathrm{MB} 083 \mathrm{C}}$. Dashed white line indicates mushroom bodies. Inhibition of $\mathrm{MBON}^{\mathrm{MB} 083 \mathrm{C}}$ with shits increase arousability in response to vibrations, while activation with TrpA1 suppresses arousability. (i) $\mathrm{CCHa} 1$ levels in the gut increase in response to dietary protein and target a sparse group of dopaminergic neurons in the brain to decrease arousability. (j) A model for adjustment of sensory gating by gut-brain interactions. In all panels, mean and S.E.M. are depicted; sample sizes and statistical analyses are shown in Extended Data Table 2. Scale bars: $100 \mu \mathrm{m}$. 


\section{EXPERIMENTAL PROCEDURES}

\section{Fly stocks}

337 Flies were grown in 12 hour light: 12 hour dark cycles at $25{ }^{\circ} \mathrm{C}$ unless indicated otherwise.

338 Experiments were performed in Percival Scientific Inc. (DR36VL model) incubators. Stocks used 339 include: wild type iso31, elav-Gal4, UAS-TrpA1 and UAS-Shit (gift from Kyunghee Koh), elav340 Gal4;UAS-Dcr2 (gift from Nicholas Stavropoulos), PAM-Gal4 (BDSC \#41347), UAS-CCHa1-RNAi 341 (BDSC \#57562), UAS-CCHa1-RNAi \#2 (VDRC \#104074), UAS-CCHa1R-RNAi (BDSC \#51168), 342 UAS-CCHa1R-RNAi \#2 (VDRC \#103055), UAS-GFP-RNAi (BDSC \#41552), EECG-Gal4 (BDSC 343 \#49037), Tdc2-Gal4 (BDSC \#9313), vGlut-Gal4 (BDSC \#26160), MB441B-split-Gal4 (BDSC 344 \#68251), MB083-split-Gal4 (BDSC \#68287/68262), CaLexA (BDSC \#66542), UAS-nlsGFP 345 (BDSC \#4776), UAS-TH-RNAi (BDSC \#25796), UAS-Dcr2 (BDSC \#24650), Mi\{MIC\}CCHa1 ${ }^{\mathrm{MI} 09190}$ 346 (BDSC \#51261), Mi\{MIC\}CCHa1R ${ }^{M 108118}$ (BDSC \#44750), TI\{2A-GAL4\}CCHa1-R[2A-GAL4] 347 (BDSC \#84601), P\{GMR23H07-GAL4\}attP2 (BDSC \#47498), and UAS-CD8GFP, UAS-nlsLacZ, 348 TH-Gal4, ddc-Gal4 and Cha-Gal4 (gift from Michael Crickmore), Tim-Gal4 (gift from Michael 349 Young) and pros-Gal4 (gift from Norbert Perrimon). All the stocks used for behavioral experiments 350 were outcrossed at least 5 times to an isogenised (iso31) genetic background. In the case of UAS351 CCHa1-RNAi and UAS-CCHa1R-RNAi the UAS-GFP-RNAi from the same library was used as a 352 control.

354 The RNAis against CCHa1R target non-overlapping sequences: VDRC \#103055 targets CCHa1R 355 coding sequence nucleotides 16 to 333, and BDSC \#51168 targets CCHa1R coding sequence 356 nucleotides 375 to 397 . The RNAis used for $\mathrm{CCHa} 1$ target sequences that partially overlap: VDRC 357 \#104974KK targets CCHa1 coding sequence nucleotides 241 to 539 and BDSC \#57562 targets 358 CCHa1 coding sequence nucleotides 525 to 547.

360 For all the Gal4/UAS experiments, heterozygous controls were used: (UAS crossed to the Gal4 361 genetic background, and Gal4 crossed to the UAS genetic background).

\section{Fly media}

364 Flies were raised on cornmeal-agar medium ${ }^{71}$. Briefly, our regular food contained $7.3 \%$ agar 365 (Spectrum), 12.7\% yeast (MP Biomedicals), 56\% sugar (Domino) and 24\% cornmeal (Bunge). 366 These ingredients were heated until the temperature reached $97^{\circ} \mathrm{C}$ while stirring. After the 367 temperature of the solution decreased to $75^{\circ} \mathrm{C}, 2.5 \%$ Tegosept, $23.8 \mathrm{~g} / \mathrm{L}$ Ethanol (Koptic \#V1001) 368 and water to compensate for evaporation were added. 


\section{Arousal threshold screen}

371 UAS-RNAi lines were from Bloomington Drosophila Stock Center, Kyoto Stock Center of

372 Drosophila Genomics and Genetic Resources, Vienna Drosophila Resource Center and TRiP

373 library generously shared by Norbert Perrimon's laboratory. These lines were selected randomly

374 (the screen was unbiased) and crossed to flies expressing elav-Gal4. 8 males, 3-5 days old, were

375 tested in the system described below. Each dot represents the behavior of 8 flies. In our

376 experiments, the flies were exposed to low amplitude stimulation $(0.2 \mathrm{~V})$ on the first night, and high

377 amplitude stimulation (2V) the second night.

\section{Analysis of screen results}

380 For the genes whose disruption in flies caused hypo- or hyper-arousability phenotypes gene 381 ontology analysis was performed. We used the GO Term Mapper (http://go.princeton.edu/cgi382 bin/GOTermMapper) to bin individual GO categories into more general terms, to facilitate the 383 analysis. In order to normalize the different GO terms taking into account their representation in 384 the genome, for every GO term the \% of hits containing that term in our screen was normalized 385 to the $\%$ of genes in the whole genome that contain that term. The data are represented on a $\log _{10}$ 386 scale.

\section{Locomotor activity and sleep monitoring}

389 Locomotor activity was recorded using the Drosophila Activity Monitoring system (DAM3, 390 Trikinetics, Waltham, MA). Flies were individually housed in $65 \mathrm{~mm}$-long glass tubes (TriKinetics) 391 filled with $\sim 40 \mathrm{~mm}$ of cornmeal-agar food, unless otherwise indicated. This left $\sim 25 \mathrm{~mm}$ of space 392 for flies to walk back and forth in. An infrared light beam bisects this space and gets interrupted 393 when flies walk through; this is automatically scored as movement. Sleep was defined as five 394 minutes of inactivity ${ }^{1,2}$. Sleep quantification was performed with a custom MATLAB software 395 (available on github at https://github.com/CrickmoreRoguljaLabs/SleepAnalysis).

\section{Arousal threshold mechanical setup}

398 DAMs were mounted on custom platforms positioned on top of large bass loudspeakers (381 mm, 399 Delta 15 LFA, Eminence, Eminence, KY). Each speaker/platform holds up to 8 DAMs. To 400 stimulate flies, a custom program (Good Morning, available on github at 401 https://github.com/IrisTitos/ATanalysis/blob/master/Goodmorning.md) written in MATLAB (The 402 MathWorks, Natick, MA) generates a multisine signal $(40 \mathrm{~Hz}-200 \mathrm{~Hz})$ using a multifunction 
403 device (USB-6211, National Instruments, Austin, TX). The signal is fed into an amplifier (SLA-1,

404 Applied Research and Technology, Rochester, NY) connected to the speaker. Using a range of

405 frequencies should avoid frequency-specificity of the response. With this setup, the mechanical

406 vibrations of the loudspeaker translate into up and down movements of the platform on which

407 DAMs are mounted. Low power accelerometers (ADXL335, Analog Devices, Norwood, MA)

408 measure movements of individual DAMs, at different positions on the platform, to ensure that

409 vibrations are uniform across all monitors. In our experiments, 2 second vibrations were delivered

410 at random intervals every 30-40 minutes, starting $1.5 \mathrm{hrs}$ after the lights turn off (for 10 hours

411 during the night (dark phase)).

413 These data are processed in MATLAB together with locomotor data from DAMs (available on 414 github at https://github.com//risTitos/ATanalysis/blob/master/ReadDamsPullDown). Only flies

415 that were asleep (more than 5 minutes of locomotor inactivity) at the moment that the stimulus 416 was applied were considered when arousability from sleep was calculated. If locomotor activity 417 was triggered within the 3 minutes following stimulus application, the fly was considered awoken

418 by vibrations. To control for the increased chance of spontaneous awakenings in genetic 419 manipulations that cause a decrease in sleep duration or increase in sleep fragmentation, we 420 normalized for spontaneous awakenings: \% change in awoken flies $=100 *$ ( $\%$ flies that wake up 421 - \% spontaneous awakenings) / (100 - \% spontaneous awakenings). To calculate spontaneous 422 awakenings, the activity data from the same night and the same flies used to calculate the "\% 423 flies that wake up" was analyzed with a modified timestamp file in which the stimulation timings 424 are shifted by 10 minutes preceding the stimulation. Next, we performed the same analysis 425 previously done with the original timestamp; only flies that were asleep at the modified timestamp 426 were considered for analysis, and flies that produced locomotor activity within the 3 minutes 427 following the modified timestamp were considered to wake up.

429 To quantify the percentage of flies that react to stimuli during wakefulness we used the same flies 430 and timestamps used to calculate the "\% change in awoken flies" since our analysis was post431 hoc. In this case, only flies that were awake immediately before the stimulation were taken into 432 account. For those flies we calculated the average activity during the 3 minutes prior to 433 stimulation, and the average activity during the 3 minutes after the stimulation. Flies were 434 considered reacting to stimuli if the average activity after the stimulation was higher than prior to 435 it. 
437 The setup for probing arousal threshold with temperature

438 To probe arousability with temperature, we used a flat multi-beam activity monitor (DAM5M

439 Trikinetics) in which the fly-containing tubes lay horizontally. A heating pad (McMaster-Carr

440 35765K469, Elmhurst, IL) controlled by a 1/16 DIN Ramp/Soak Controller (Omega CN 7800,

441 Norwalk, CT) covers the surface of the monitor, Custom-made software (MATLAB) turns on the

442 heating pad, increasing the temperature to $40{ }^{\circ} \mathrm{C}$ (unless otherwise indicated) for 2 minutes, at

443 random intervals (30-40 minutes) during the night. The same protocol as described for the

444 mechanical setup was used to calculate the \% of flies awoken by heat.

Feeding experiments

447 Male flies, 3 to 5 days old, were transferred from regular food to different food conditions

448 approximately $10 \mathrm{hrs}$ after light turning on (i.e. CT10 in 12:12 light-dark cycles), unless otherwise 449 indicated. After 24 hours, they were dissected or examined for behavior. The cornmeal-agar food 450 was supplemented with 10\% coconut oil (Spectrum essentials \#L215562P-006), 1\% propionic 451 acid (Sigma-Aldrich \#P1386), 0.5\% hexanoic acid (Sigma-Aldrich \#P21530), $200 \mathrm{mM}$ glucose 452 (Sigma-Aldrich \#158968-1KG), 200 mM Fructose (Sigma-Aldrich \#F0127), 200 mM Galactose 453 (Sigma-Aldrich \#G0750), 10\% peptone (Apex \#20-260), and the single amino acids (Sigma454 Aldrich LAA21-1KT) or water as a control.

\section{Food intake measurement}

457 3-5 day old male flies were transferred from regular food to regular food, or to regular food 458 supplemented with peptone containing 2\% (wt/vol) FD\&C Blue \#1 (Spectrum \#FD110). After 24 459 hours, flies were frozen at $-20{ }^{\circ} \mathrm{C}$. Frozen flies were decapitated (to avoid eye the pigment 460 interfering with the measurement), aliquoted in groups of 5 into Eppendorf tubes containing $50 \mu \mathrm{L}$ 461 of $1 \%$ Triton X-100 1X-PBS and homogenized with a motorized pestle (Argos \#9950-901). 462 Samples were centrifuged and supernatant was measured at $660 \mathrm{~nm}$ (A660) in a NanoDrop 2000 463 Spectrophotometer (Thermo Fisher Scientific). A standard curve was generated using serial 464 dilutions of the blue dye and the sample dye concentration was extrapolated from that curve.

\section{Immunohistochemistry}

467 Adult males were anesthetized with carbon dioxide, washed briefly with $70 \%$ ethanol (Koptic 468 \#V1001) and dissected in 1X-PBS (Phosphate Buffered Saline 10X SeraCare \#5460-0030). Guts 469 were fixed overnight at $4{ }^{\circ} \mathrm{C}$ in PBS with $4 \%$ paraformaldehyde (PFA, Electron Microscopy 470 Sciences \#15710). Dissected brains and ventral nervous systems were fixed for 30 minutes in 
4\% PFA at room temperature. Tissues were washed 3 times for 20 minutes in 1X-PBS containing 0.2\% Triton X-100 (Amresco \#M143), and then blocked for 2 hours in 1X PBS, 0.2\%Triton X-100,

$4732 \%$ bovine serum albumin (BSA Gemini \#700-101P) at room temperature. Samples were 474 incubated with primary antibodies overnight (except for Bruchpilot (Brp) stainings, which were 475 incubated for $\sim 48$ hours at $4{ }^{\circ} \mathrm{C}$ in $1 \mathrm{X}$ PBS, $0.2 \%$ Triton $\mathrm{X}-100,2 \%$ BSA at the indicated dilutions).

476 Tissues were washed 3 times for 20 minutes in $1 X-P B S, 0.2 \%$ Triton $X-100$ and then incubated 477 with secondary antibodies for 2 hours at room temperature at the indicated dilutions (except for 478 Bruchpilot stainings that were incubated for $\sim 48$ hours at $4{ }^{\circ} \mathrm{C}$ ). Samples were then washed 3 479 times for 20 minutes in 1X-PBS, 0.2\% Triton X-100, and mounted in-between glass slides and 480 coverslips (Electron Microscopy Sciences \#72230-01 and 64321-10) in Prolong Gold Antifade 481 medium (Invitrogen \#1942345).

483 Rabbit anti-CCHa1 antibodies were raised against the peptide QIDADNENYSGYELT ${ }^{21}$ by 484 Genscript and affinity purified by the company.

Primary antibodies used: mouse anti-Bruchpilot (1:7, DSHB nc82), rabbit anti-CCHa1 (1:50), chicken anti-GFP (1:1000, Aves \#GFP-1020), mouse anti-prospero (1:50, DSHB pros) and mouse anti-tyrosine hydroxylase (TH) (1:1000 ImmunoStar \#22941).

Secondary antibodies used: Alexa Fluor 488 goat anti-chicken (1:1000 Life technologies \#A11039), Alexa Fluor 488 goat anti-rabbit (1:1000 Life technologies \#A11034), Alexa Fluor 647 goat anti-rabbit (1:1000 Life technologies \#A21244) Alexa Fluor 647 donkey anti-mouse (1:1000

493 Life technologies \#A31571).

\section{Fluorescence microscopy}

496 Fluorescent images were acquired with a Leica SP8 confocal microscope. All the imaging 497 conditions remained constant within each experiment, only the $Z$ start and end settings were 498 adjusted individually for each sample. The average distance between Z-stacks was 100 microns.

\section{Image quantification}

501 Quantification of CCHa1 and GFP signals was performed with Fiji. For every image, a Z-stack 502 summation projection of the whole gut was obtained. To quantify $\mathrm{CCHa} 1$ antibody staining in the 503 gut, $\mathrm{CCHa} 1$ signal was used to define the region of interest (ROI). The average pixel intensity 504 and area of that ROI was measured. A region with similar area adjacent to the ROI was selected 
505 to measure the average background pixel intensity. The amount of CCHa1 was quantified by

506 subtracting the background from the $\mathrm{CCHa} 1$ signal and multiplying by the $\mathrm{CCHa} 1 \mathrm{ROI}$ area.

508 For Fig. 3c and Extended Data Fig. 6a, the percentage of activated CCHa1 cells in the gut was

509 calculated based on CaLexA-dependent GFP transcription. First, we quantified how many

$510 \mathrm{CCHa1-expressing} \mathrm{cells} \mathrm{were} \mathrm{present} \mathrm{in} \mathrm{the} \mathrm{posterior} \mathrm{midgut} \mathrm{using} \mathrm{the} \mathrm{"counter"} \mathrm{function} \mathrm{of} \mathrm{Fiji.}$

511 Then, we counted how many of those cells also have GFP signal. To determine the \% of $\mathrm{CCHa} 1$

512 cells that were activated, we used the formula: (CCHa1 and GFP-positive cells/all CCHa1-positive

513 cells)*100.

515 To quantify GFP intensity in Fig. 4c,g and Extended Data Fig. 9c, we projected the summation of 516 the Z-stacks containing the cells of interest. Each cell was manually selected and the average 517 pixel intensity of the GFP signal was quantified. A region next to the cells of interest but containing 518 no GFP positive cells was used to measure the background signal. To calculate the GFP signal 519 we used this formula: (GFP average pixel intensity from the cell - GFP background average pixel 520 intensity)

\section{Quantitative RT-PCR}

523 For each condition, 2 whole flies, 5 brains or 10 guts of 3-5 days old males were dissected and 524 immediately transferred into $100 \mu$ of ice-cold TRIzol (Thermo fisher \#15596026) one by one, 525 flash-frozen on dry ice and stored at $-80^{\circ} \mathrm{C}$. To extract RNA, samples were homogenized with a 526 pestle (Argos \#9950-901) for 20 seconds on ice before adding $200 \mu \mathrm{l}$ of TRIzol and $60 \mu \mathrm{l}$ of 527 chloroform. Samples were then centrifuged for 15 minutes at $15000 \mathrm{rpm} 4{ }^{\circ} \mathrm{C}$. The supernatant 528 was collected and diluted in an equal amount of ethanol 99\% (Koptec \#V1001), mixed and 529 transferred to a purification column (Zymo kit Direct-zol R2061). The manufacturer's instructions 530 for RNA purification and subsequent DNAse treatment were followed (DNAse Turbo DNA-free kit 531 AM1907). RNA was quantified using NanoDrop. $500 \mathrm{ng}$ of RNA were used for the 532 retrotranscriptase reaction (Biorad iScript cDNA Synthesis \#1708890). DNA product was diluted 533 1:10 and used to set up quantitative PCR reactions using Sybr Green (Biorad \#170-8880). 534 Primers were obtained from ID Technologies:

535 CCHa1 (F-ACTGACGTCGGACAATTTGC and R-ACACGAATGTCCGTATTCCA) ${ }^{13}$

536 CCHa1R (F-GTTCCAAACACCTACATTTTATCAC and R-CGGATAATGCAGTCAGCGTA) ${ }^{13}$

537 CCHa2 (F-AAACAGCAACAGCAGCAAAC and R- AGGACCACGGTGCAGATAAC) ${ }^{13}$

538 CCHa2R (F-CATACCCAACACATACATTCTTTC and R- GAAAGGGCGGTCAGTGTAAA) ${ }^{13}$ 
Conditional experiments

542 Adult-specific knockdown of $\mathrm{CCHa} 1$ or its receptor was achieved using a temperature-sensitive

543 allele of Gal80, a Gal4 inhibitor $\left(\right.$ Gal80 $\left.{ }^{\text {ts }}\right)$. Flies were raised at $21^{\circ} \mathrm{C}$, temperature at which Gal80 ${ }^{\text {ts }}$

544 is stable and interferes with Gal4 function. Two days after eclosion, temperature was raised to 29

$545{ }^{\circ} \mathrm{C}$, temperature at which $\mathrm{Gal} 80^{\text {ts }}$ is degraded and the Gal4 driver becomes functional.

547 For TrpA1 and shits experiments, flies were raised at $21^{\circ} \mathrm{C}$, temperature at which $\operatorname{TrpA} 1$ and shi 548 are not active. Flies were transferred to $27^{\circ} \mathrm{C}$ to analyze behavior. In Figures 2d, Extended Data 549 Figure 4b, and Extended Data Figure 5, the experiment needs to be done at least at $25^{\circ} \mathrm{C}$ to 550 ensure CCHa1-RNAi expression, since the Gal4-UAS system depends on temperature (Gal4 551 expression is higher at higher temperatures). TrpA1 has been reported to be active at 552 temperatures above $25^{\circ} \mathrm{C}$, as low as $26^{\circ} \mathrm{C}^{12}$. We cannot completely discard that some mild TrpA1

553 activation is occurring at $25^{\circ} \mathrm{C}$. However, it does not seem to be enough to cause behavioral 554 phenotype since we do not observe any arousal threshold phenotypes at $25^{\circ} \mathrm{C}$ when EECG-Gal4 555 drives $\operatorname{TrpA} 1$, while arousal is strongly decreased at $27^{\circ} \mathrm{C}$.

\section{Statistical analysis}

558 Statistical analyses were performed using GraphPad Prism software 7 (GraphPad Software Inc.,

559 San Diego, CA). Except for the screen data, all experiments were done 3 times independently.

560 Data was tested for normality and all the statistical tests were two-sided All data are presented 561 as mean \pm S.E.M. Please see Table $S 1$ for sample sizes, tests and $P$ values.

\section{DATA AND SOFTWARE AVAILABILITY}

564 All data and materials are available upon request. 


\section{REFERENCES}

568 1. Hendricks, J. C. et al. Rest in Drosophila is a sleep-like state. Neuron 25, 129-38 (2000).

569 2. Shaw, P. J., Cirelli, C., Greenspan, R. J. \& Tononi, G. Correlates of sleep and waking in

570 Drosophila melanogaster. Science 287, 1834-7 (2000).

571 3. Tomita, J., Ban, G. \& Kume, K. Genes and neural circuits for sleep of the fruit fly.

$572 \quad$ Neurosci Res 118, 82-91 (2017).

573 4. Lebestky, T. et al. Two different forms of arousal in Drosophila are oppositely regulated

574 by the dopamine D1 receptor ortholog DopR via distinct neural circuits. Neuron 64, 522-

$575 \quad 536(2009)$.

576 5. Ferguson, L. et al. Transient Dysregulation of Dopamine Signaling in a Developing

577 Drosophila Arousal Circuit Permanently Impairs Behavioral Responsiveness in Adults.

$578 \quad$ Front Psychiatry 8, 22 (2017).

579 6. Donlea, J. M. et al. Recurrent Circuitry for Balancing Sleep Need and Sleep. Neuron 97, $580 \quad 378-389$ e4 (2018).

581 7. Troup, M. et al. Acute control of the sleep switch in Drosophila reveals a role for gap junctions in regulating behavioral responsiveness. Elife 7, (2018).

8. Chen, K. F., Lowe, S., Lamaze, A., Kratschmer, P. \& Jepson, J. Neurocalcin regulates nighttime sleep and arousal in Drosophila. Elife 8, (2019).

9. Van Someren, E. J. et al. Disrupted Sleep: From Molecules to Cognition. J Neurosci 35, 13889-13895 (2015).

10. Lin, D. M. \& Goodman, C. S. Ectopic and increased expression of fasciclin II alters motoneuron growth cone guidance. Neuron 13, 507-523 (1994).

12. Farhan, A. et al. The CCHamide 1 receptor modulates sensory perception and olfactory

11. Ida, T. et al. Isolation of the bioactive peptides CCHamide-1 and CCHamide-2 from

594 13. Li, S. et al. Expression patterns of the Drosophila neuropeptide CCHamide-2 and its receptor may suggest hormonal signaling from the gut to the brain. PLoS One 8, e76131 (2013).

597 14. Sano, H. et al. The Nutrient-Responsive Hormone CCHamide-2 Controls Growth by 
Regulating Insulin-like Peptides in the Brain of Drosophila melanogaster. PLoS Genet 11, e1005209 (2015).

600 15. Ren, G. R. et al. CCHamide-2 Is an Orexigenic Brain-Gut Peptide in Drosophila. PLoS One 10, e0133017 (2015).

602 16. Fujiwara, Y. et al. The CCHamide1 Neuropeptide Expressed in the Anterior Dorsal Neuron 1 Conveys a Circadian Signal to the Ventral Lateral Neurons in Drosophila melanogaster. Front. Physiol. 9, 1276 (2018).

17. Pagani, M. et al. How Gastrin-Releasing Peptide Opens the Spinal Gate for Itch. Neuron 103, 102-117 e5 (2019).

18. Yamada, K., Wada, E. \& Wada, K. Male mice lacking the gastrin-releasing peptide receptor (GRP-R) display elevated preference for conspecific odors and increased social investigatory behaviors. Brain Res. 870, 20-26 (2000).

19. Yamada, K., Wada, E. \& Wada, K. Bombesin-like peptides: studies on food intake and social behaviour with receptor knock-out mice. Ann. Med. 32, 519-529 (2000).

20. Nagarkar-Jaiswal, S. et al. A library of MiMICs allows tagging of genes and reversible, spatial and temporal knockdown of proteins in Drosophila. Elife 4, (2015).

21. Veenstra, J. A. \& Ida, T. More Drosophila enteroendocrine peptides: Orcokinin B and the CCHamides 1 and 2. Cell Tissue Res. 357, 607-21 (2014).

22. Miguel-Aliaga, I., Jasper, H. \& Lemaitre, B. Anatomy and Physiology of the Digestive Tract of Drosophila melanogaster. Genetics 210, 357-396 (2018).

23. Song, W., Veenstra, J. A. \& Perrimon, N. Control of lipid metabolism by tachykinin in Drosophila. Cell Rep 9, 40-47 (2014).

24. Sen, A., Kuruvilla, D., Pinto, L., Sarin, A. \& Rodrigues, V. Programmed cell death and context dependent activation of the EGF pathway regulate gliogenesis in the Drosophila

26. Park, J.-H. et al. A subset of enteroendocrine cells is activated by amino acids in the Drosophila midgut. FEBS Lett. 590, 493-500 (2016).

627 27. Waddell, S., Armstrong, J. D., Kitamoto, T., Kaiser, K. \& Quinn, W. G. The amnesiac 
Memory. Cell 103, 805-813 (2000).

28. Hamada, F. N. et al. An internal thermal sensor controlling temperature preference in Drosophila. Nature 454, 217-220 (2008).

29. Scopelliti, A. et al. A Neuronal Relay Mediates a Nutrient Responsive Gut/Fat Body Axis Regulating Energy Homeostasis in Adult Drosophila. Cell Metab 29, 269-284 e10 (2019).

30. Chen, J. et al. Allatostatin A Signalling in Drosophila Regulates Feeding and Sleep and Is Modulated by PDF. PLoS Genet 12, e1006346 (2016).

31. Murphy, K. R. et al. Postprandial sleep mechanics in Drosophila. Elife 5, (2016).

32. Yurgel, M. E. et al. A single pair of leucokinin neurons are modulated by feeding state and regulate sleep-metabolism interactions. PLoS Biol 17, e2006409 (2019).

33. Masuyama, K., Zhang, Y., Rao, Y. \& Wang, J. W. Mapping Neural Circuits with Activity-Dependent Nuclear Import of a Transcription Factor. J. Neurogenet. 26, 89-102 (2012).

34. Liu, C. et al. A subset of dopamine neurons signals reward for odour memory in Drosophila. Nature 488, 512-516 (2012).

35. Aso, Y. et al. The neuronal architecture of the mushroom body provides a logic for associative learning. Elife 3, e04577 (2014).

36. Sitaraman, D., Aso, Y., Rubin, G. M. \& Nitabach, M. N. Control of Sleep by (2015).

37. Landayan, D., Feldman, D. S. \& Wolf, F. W. Satiation state-dependent dopaminergic control of foraging in Drosophila. Sci. Rep. 8, 5777 (2018).

38. Aso, Y. et al. Mushroom body output neurons encode valence and guide memory-based action selection in Drosophila. Elife 3, e04580 (2014).

39. Aso, Y. et al. Nitric oxide acts as a cotransmitter in a subset of dopaminergic neurons to diversify memory dynamics. Elife 8, (2019).

40. Kume, K., Kume, S., Park, S. K., Hirsh, J. \& Jackson, F. R. Dopamine is a regulator of

657 41. Riemensperger, T. et al. Behavioral consequences of dopamine deficiency in the Drosophila central nervous system. Proc Natl Acad Sci U S A 108, 834-839 (2011). 
a miniature brain. Proc. R. Soc. B Biol. Sci. 278, 906-913 (2011).

43. Wisor, J. P. Dopamine and Wakefulness: Pharmacology, Genetics, and Circuitry. Handb Exp Pharmacol 253, 321-335 (2019).

44. Heisenberg, M., Borst, A., Wagner, S. \& Byers, D. Drosophila mushroom body mutants are deficient in olfactory learning. J Neurogenet 2, 1-30 (1985).

45. de Belle, J. S. \& Heisenberg, M. Associative odor learning in Drosophila abolished by chemical ablation of mushroom bodies. Science (80-. ). 263, 692-695 (1994).

667 46. Strausfeld, N. J., Hansen, L., Li, Y., Gomez, R. S. \& Ito, K. Evolution, Discovery, and Interpretations of Arthropod Mushroom Bodies. Learn. Mem. 5, 11-37 (1998).

47. Liu, L., Wolf, R., Ernst, R. \& Heisenberg, M. Context generalization in Drosophila visual learning requires the mushroom bodies. Nature 400, 753-756 (1999).

48. Hong, S. T. et al. cAMP signalling in mushroom bodies modulates temperature preference behaviour in Drosophila. Nature 454, 771-775 (2008).

673 49. Vogt, K. et al. Shared mushroom body circuits underlie visual and olfactory memories in Drosophila. Elife 3, e02395 (2014).

675 50. Martin, J. R., Ernst, R. \& Heisenberg, M. Mushroom bodies suppress locomotor activity 676 in Drosophila melanogaster. Learn Mem 5, 179-191 (1998).

677 51. Dubnau, J., Grady, L., Kitamoto, T. \& Tully, T. Disruption of neurotransmission in Drosophila mushroom body blocks retrieval but not acquisition of memory. Nature 411,

680 52. McGuire, S. E., Le, P. T. \& Davis, R. L. The role of Drosophila mushroom body signaling in olfactory memory. Science (80-. ). 293, 1330-1333 (2001).

682 53. Joiner, W. J., Crocker, A., White, B. H. \& Sehgal, A. Sleep in Drosophila is regulated by adult mushroom bodies. Nature 441, 757-760 (2006).

684 54. Pitman, J. L., McGill, J. J., Keegan, K. P. \& Allada, R. A dynamic role for the mushroom 685 bodies in promoting sleep in Drosophila. Nature 441, 753-756 (2006).

686 55. Zhang, K., Guo, J. Z., Peng, Y., Xi, W. \& Guo, A. Dopamine-mushroom body circuit regulates saliency-based decision-making in Drosophila. Science (80-. ). 316, 1901-1904 (2007).

689 56. Cohn, R., Morantte, I. \& Ruta, V. Coordinated and Compartmentalized Neuromodulation 
691 57. Heisenberg, M. Mushroom body memoir: from maps to models. Nat Rev Neurosci 4, 266$692 \quad 275(2003)$.

693 58. Eschbach, C. et al. Recurrent architecture for adaptive regulation of learning in the insect 694 brain. Nat. Neurosci. 23, 544-555 (2020).

695 59. Dolan, M.-J. et al. Neurogenetic dissection of the Drosophila lateral horn reveals major outputs, diverse behavioural functions, and interactions with the mushroom body. Elife $\mathbf{8}$, (2019).

60. He, L., Binari, R., Huang, J., Falo-Sanjuan, J. \& Perrimon, N. In vivo study of gene expression with an enhanced dual-color fluorescent transcriptional timer. Elife 8, (2019).

61. Campbell, S. S. \& Tobler, I. Animal sleep: A review of sleep duration across phylogeny. Neurosci. Biobehav. Rev. 8, 269-300 (1984).

62. Huda, R., Goard, M. J., Pho, G. N. \& Sur, M. Neural mechanisms of sensorimotor transformation and action selection. Eur. J. Neurosci. (2018) doi:10.1111/ejn.14069.

63. Murakami, M., Kashiwadani, H., Kirino, Y. \& Mori, K. State-Dependent Sensory Gating in Olfactory Cortex. Neuron 46, 285-296 (2005).

64. Eban-Rothschild, A., Appelbaum, L. \& de Lecea, L. Neuronal Mechanisms for Sleep/Wake Regulation and Modulatory Drive. Neuropsychopharmacology 43, 937-952 (2018).

65. Zhang, S. X., Rogulja, D. \& Crickmore, M. A. Recurrent Circuitry Sustains Drosophila Courtship Drive While Priming Itself for Satiety. Curr Biol 29, 3216-3228 e9 (2019).

66. Zhang, S. X., Rogulja, D. \& Crickmore, M. A. Dopaminergic Circuitry Underlying

713 67. van den Pol, A. N. Neuropeptide transmission in brain circuits. Neuron 76, 98-115 (2012).

715 68. Fernandes, A. B. et al. Postingestive Modulation of Food Seeking Depends on Vagus-

716 Mediated Dopamine Neuron Activity. Neuron 106, 778-788.e6 (2020).

717 69. Rao, M. \& Gershon, M. D. The bowel and beyond: the enteric nervous system in neurological disorders. Nat Rev Gastroenterol Hepatol 13, 517-528 (2016).

719 70. Valeur, J. Gut-brain axis in history and culture. Microb. Ecol. Health Dis. 29, (2019).

720 71. Vaccaro, A. et al. Sleep Loss Can Cause Death through Accumulation of Reactive Oxygen Species in the Gut. Cell 181, 1307-1328.e15 (2020). 


\section{EXTENDED DATA}

Extended Data Figure 1. Experimental setup and analysis of the genes that regulate arousability, uncovered in our screen. (a) Picture of the setup used for mechanical stimulation. Custom software generates 2-second-long multi-sine $(40-200 \mathrm{~Hz})$ signals that are converted into mechanical stimuli (vibrations). Vibrations are given at random intervals centered on 30-40 minutes throughout the night. DAM monitors are mounted on custom-built platforms on top of the loudspeakers. Vibrations produced by the loudspeaker are translated into vibrations of the DAMs. Accelerometers placed on top of the DAMs measure the stimulus intensities, to ensure that all flies are stimulated equally regardless of their position on the platform. (b) Each fly is housed in a glass tube, with $\sim 25 \mathrm{~mm}$ of space for movement. Movement is detected by the interruption of the infra-red light beam and recorded every minute using the DAM system (Trikinetics, Waltham, MA). (c) Analysis of the gene ontology terms for Biological process, Molecular function and Cellular component for the genes whose disruption produced hypo- or hyper-arousability phenotypes. We used the GO Term Mapper (http://go.princeton.edu/cgi-bin/ GOTermMapper) to bin individual GO categories into more general terms, to facilitate analysis. We represent a GO term enrichment in our screen results compared to the relative weight of that GO term in the whole genome. To do this, we calculated the \% of hits in our screen that had each GO term and normalized to the $\%$ of genes in the whole genome that contain that term. The data are represented on a $\log _{10}$ scale.

Extended Data Figure 2. CCHa1 and CCHa1R mutants and RNAi lines. (a) elav-Gal4-driven RNAi against $\mathrm{CCHa} 1$ (elav $>\mathrm{CCHa} 1-\mathrm{RNAi}$ ) or its receptor (elav $>\mathrm{CCHa} 1 \mathrm{R}-\mathrm{RNAi}$ ) increases arousal in awake flies. elav $>\mathrm{CCHa1-RNAi}$ also causes fragmented sleep (number of sleep episodes is slightly increased, while their duration is decreased); elav $>$ CCHa1R-RNAi decreases total sleep duration due to a decrease in sleep bout length. The last panel shows sleep amount throughout the day, in 30-minute intervals. The white box at the top indicates day period (lights on) while black box indicates night period (lights off). (b) qRT-PCR shows that elav>CCHa1-RNAi depletes $\mathrm{CCHa} 1$ in the whole fly while it does not affect $\mathrm{CCHa}$. Each dot represents a sample of 2 whole flies. CCHa1R but not $\mathrm{CCHa} 2 \mathrm{R}$ is depleted in fly brains when CCHa1R-RNAi is driven by elav-Gal4. Each dot represents a sample of 5 brains. (c) Arousal during sleep, total sleep duration, arousal during wakefulness, and basal locomotor activity data for additional CCHa1and CCHa1R-RNAi lines. (d) qRT-PCR shows that elav>CCHa1-RNAi\#2 depletes CCHa1 in the whole fly while it does not affect $\mathrm{CCHa} 2$. Each dot represents a sample of 2 whole flies. CCHa1R but not $\mathrm{CCHa} 2 \mathrm{R}$ is depleted in fly brains when $\mathrm{CCHa1R-RNAi \# 2} \mathrm{is} \mathrm{driven} \mathrm{by} \mathrm{elav-Gal4.} \mathrm{Each} \mathrm{dot}$ represents a sample of 5 brains. (e) Mutants for $\mathrm{CCHa} 1$ or its receptor increase arousability during wakefulness and reduce total sleep amount. The last panel shows sleep amount throughout the day in 30-minute intervals. The white box at the top indicates day period (lights on), the black box indicates night period (lights off). (f) Conditional, adult-specific knockdown of CCHa1 or its receptor was achieved using a temperature-sensitive allele of Gal80, a Gal4 inhibitor (elavGal4;Tub-Gal80 ${ }^{\text {ts }}$ ). Flies were raised at $21{ }^{\circ} \mathrm{C}$, temperature at which $\mathrm{Gal} 80^{\text {ts }}$ is stable and interferes with Gal4 function. Two days after eclosion, temperature was raised to $29{ }^{\circ} \mathrm{C}$, temperature at which $\mathrm{Gal} 80^{\text {ts }}$ is degraded and the Gal4 driver becomes functional. Conditional knockdown phenocopied the results seen with elav-Gal4. In all panels, mean and S.E.M. are depicted; sample sizes and statistical analyses are in Extended Data Table 2.

768 

duration. (a) Mi\{MIC\}CCHa1 ${ }^{\text {MI09190 }}$ contains the GFP open reading frame inserted downstream of the $\mathrm{CCHa} 15$ ' UTR. Heterozygotes for the Mi\{MIC\}CCHa1 ${ }^{\text {Mlo9190 }}$ construct show a GFP pattern of expression that overlaps with the $\mathrm{CCHa} 1$ antibody. Homozygotes for Mi\{MIC\}CCHa $1^{\mathrm{M} 109190}$ mutation show the same GFP expression pattern as heterozygotes but do not show any $\mathrm{CCHa} 1$ antibody signal since both copies of the gene are mutated. (b) nls::GFP expression pattern driven by EECG-Gal4 and pros-Gal4 in the central nervous system shows no co-localization with antibodies against $\mathrm{CCHa} 1$. (c) $\mathrm{CCHa} 1$ in the gut co-localizes with prospero, a marker of enteroendocrine cells. (d) elav-Gal4-driven nlsLacZ shows that the driver labels CCHa1producing enteroendocrine cells in the posterior midgut. (e) $\mathrm{CCHa} 1$ signal in the gut is abolished when CCHa1-RNAi is expressed with elav-Gal4, EECG-Gal4, or pros-Gal4:TubGal80 ts. Rectangles indicate the regions shown in Fig. 2a. (f) When CCHa1 is knocked down using EECGGal4, responsiveness in awake flies is increased but there are no changes in basal locomotion, sleep duration, sleep bout number and length. The last panel shows sleep amount throughout the day, in 30-minute intervals. The white box at the top indicates day period (lights on) while black box indicates night period (lights off). (g) When CCHa1 is knocked down using prosGal4:TubGal $80^{\text {ts }}$, responsiveness in awake flies is increased but there are no changes in basal locomotion, sleep duration, sleep bout number and length. The last panel shows sleep amount throughout the day, in 30-minute intervals. The white box at the top indicates day period (lights on) while black box indicates night period (lights off). (h) Knocking down $\mathrm{CCHa} 1$ in the gut does not affect the feeding behavior of flies as measured with blue dye. Each dot represents the average of three experiments, each done with 10 flies. In all panels, mean and S.E.M. are depicted; sample sizes and statistical analyses are in Extended Data Table 2. Scale bars: 100 $\mu \mathrm{m}$.

Extended Data Figure 4. Enteroendocrine cells (labeled with EECG-Gal4 driver) regulate arousability through $\mathrm{CCHa} 1$. (a) Inactivation of $\mathrm{CCHa} 1$-expressing cells in the gut (EECG>shits) increases responsiveness in awake flies but does not affect basal locomotion, sleep duration, sleep bout number and length. The last panel shows sleep amount throughout the day, in 30minute intervals. The white box at the top indicates day period (lights on) while black box indicates night period (lights off). (b) Activation of CCHa1-expressing cells in the gut (EECG>TrpA1) decreases responsiveness in awake animals in a $\mathrm{CCH} 1$-dependent manner, and decreases sleep independently of $\mathrm{CCHa} 1$. The experiment needs to be done at least at $25{ }^{\circ} \mathrm{C}$ to ensure CCHa1-RNAi expression (see methods). The last panel shows sleep amount throughout the day, in 30-minute intervals. The white box at the top indicates day period (lights on) while black box indicates night period (lights off). The statistical significances between the experimental and its two parental controls at the same temperature are shown in the figure. In all panels, mean and S.E.M. are depicted; sample sizes and statistical analyses are in Extended Data Table 2.

\section{Extended Data Figure 5. Enteroendocrine cells (labeled with pros-Gal4;TubGal80 ${ }^{\text {ts }}$ driver)} regulate arousability through $\mathrm{CCHa} 1$. Activation of $\mathrm{CCHa} 1$-expressing cells in the gut (prosGal4;TubGal80 ${ }^{\text {ts }}>$ TrpA1) decreases responsiveness in awake animals in a CCHa1-dependent manner, and decreases sleep. The experiment needs to be done at least at $25{ }^{\circ} \mathrm{C}$ to ensure CCHa1-RNAi expression (see methods). The last panel shows sleep amount throughout the day, in 30-minute intervals. The white box at the top indicates day period (lights on) while black box indicates night period (lights off). The statistical significances between the experimental genotypes and their parental controls at the same temperature is shown in the figure. In all panels, mean and S.E.M. are depicted; sample sizes and statistical analyses are in Extended Data Table

818 2. 

Supplementing regular food with other sources of sugar (galactose and fructose) or fat (propionic acid or hexanoic acid) does not activate enteroendocrine cells or increase $\mathrm{CCHa} 1$ protein levels. (b) Access to peptone-supplemented food leads to $\mathrm{CCH} 1$ increase in the gut. The longer the flies are fed peptone, the higher the CCHa1 levels. (c) Supplementation with a mix of amino acids phenocopies the effect of peptone on arousal. Supplementation with individual amino acids or biochemically-defined amino acid groups does not recapitulate the effect of peptone on CCHa1levels in the gut; total amino acid concentration is lower in this case. (d) Plot of the same $\mathrm{CCHa} 1$ signal in the gut shown in (c), according to the amount of amino acid added. (e) Peptone supplementation slightly increases sleep, decreases responsiveness to stimulation during wakefulness and slightly reduces basal locomotion. Sleep bout number or length are not affected by peptone supplementation. The last panel shows sleep amount throughout the day in 30-minute intervals, The white box at the top indicates day period (lights on) while black box indicates night period (lights off). (f) Supplementing regular food with peptone does not affect the feeding behavior of flies, as reported by quantification of blue dye ingested with the food. Each dot represents the average of three experiments, each done with 10 flies. (g) Knocking down CCHa1 specifically in the gut with EECG-Gal4 or pros-Gal4:TubGal80 ${ }^{\text {ts }}$ diminishes the peptone-induced changes in arousal during wakefulness. In all panels, mean and S.E.M. are depicted; sample sizes and statistical analyses are in Extended Data Table 2.

Extended Data Figure 7. $\mathrm{CCHa} 1$ regulates arousal through dopaminergic neurons in the brain. (a) From the indicated Gal4 drivers, only elav-Gal4 produces a phenotype when used to knock down $\mathrm{CCH} 1$ receptor. (b) Responsiveness in awake flies, basal locomotor activity and total daily sleep duration when the CCHa1 receptor is knocked down with Ddc-Gal4 or PAM-Gal4. (c) Two different Gal4s expressed under the CCHa1R regulatory elements (P\{GMR23H07GAL4\}attP2 and TI\{2A-Gal4\}CCHa1-R2A-Gal4) show expression in the PAM cluster of dopaminergic neurons. (d) PAM ${ }^{M B 441 B}$ driver shows no expression in the gut. (e) Knocking down $\mathrm{CCHa} 1$ receptor in the $\mathrm{PAM}^{\mathrm{MB} 441 \mathrm{~B}}$ dopaminergic neurons does not affect arousal during wakefulness, sleep duration, sleep bout duration or sleep bout number, but it reduces basal locomotion. The last panel shows sleep amount throughout the day, in 30-minute intervals. The white box at the top indicates day period (lights on) while black box indicates night period (lights off). (f) Knocking down CCHa1R in PAM ${ }^{\mathrm{MB} 441 \mathrm{~B}}$ neurons does not affect feeding behavior as reported by quantification of blue dye ingested with the food. Each dot represents the average of three experiments, each done with 10 flies. (g) Knocking down CCHa1R in PAM ${ }^{\text {MB441B }}$ neurons does not alter the peptone-induced changes in arousal during wakefulness. In all panels, mean and S.E.M. are depicted; sample sizes and statistical analyses are in Extended Data Table 2.

\section{Extended Data Figure 8. PAM ${ }^{\mathrm{MB} 441 \mathrm{~B}}$ neurons regulate arousal through dopamine. (a)} Activation or inhibition of PAM ${ }^{\mathrm{MB} 441 \mathrm{~B}}$ neurons does not affect arousability during wakefulness, sleep duration, basal locomotion, sleep bout number or sleep bout length. The last panel shows sleep amount throughout the day, in 30-minute intervals. The white box at the top indicates day period (lights on) while black box indicates night period (lights off). (b) Knocking down TH in $\mathrm{PAM}^{\mathrm{MB} 441 \mathrm{~B}}$ neurons does not change arousability in awake flies, sleep duration, basal locomotor activity, sleep bout number or sleep bout length. The last panel shows sleep amount throughout the day, in 30-minute intervals. The white box at the top indicates day period (lights on) while black box indicates night period (lights off). In all panels, mean and S.E.M. are depicted; sample sizes and statistical analyses are in Extended Data Table 2. 
Extended Data Figure 9. MBONs integrate information relevant for sleep depth and duration. (a) Picture of the setup used for thermal stimulation. The percentage of flies that wake up increases as the stimulating temperature goes up $\left(35 \mathrm{vs} 40^{\circ} \mathrm{C}\right)$. (b) Responsiveness to heating during sleep or wakefulness is unaffected by peptone feeding, $\mathrm{CCHa} 1$ knockdown in the gut, or CCHa1R knockdown in $\mathrm{PAM}^{\mathrm{MB} 441 \mathrm{~B}}$ cells. (c) Thermal stimulation does not affect the activity of $\mathrm{PAM}^{\mathrm{MB} 441 \mathrm{~B}}$ neurons, as visualized with a calcium-dependent GFP reporter CaLexA. (d) MBON ${ }^{\mathrm{MB} 083 \mathrm{C}}-\mathrm{Gal} 4$ driver shows no expression in the fly gut. (e) Arousability during wakefulness, sleep duration, basal locomotion or sleep bout length are not altered by changing the activity of MBON ${ }^{\mathrm{MB} 083 \mathrm{C}}$ neurons. Inhibition and activation of $\mathrm{MBON}^{\mathrm{MB} 083 \mathrm{C}}$ slightly increase and decrease sleep bout length, respectively. The last panel shows sleep amount throughout the day, in 30minute intervals. The white box at the top indicates day period (lights on) while black box indicates night period (lights off). In all panels, mean and S.E.M. are depicted; sample sizes and statistical analyses are in Extended Data Table 2.

\section{Extended Data Table 1. List of genes from the screen that when knocked down using elav-Gal4 show hypo or hyper-arousable phenotypes}

\section{Extended Data Table 2. Sample sizes and statistical analysis}


Central nervous system
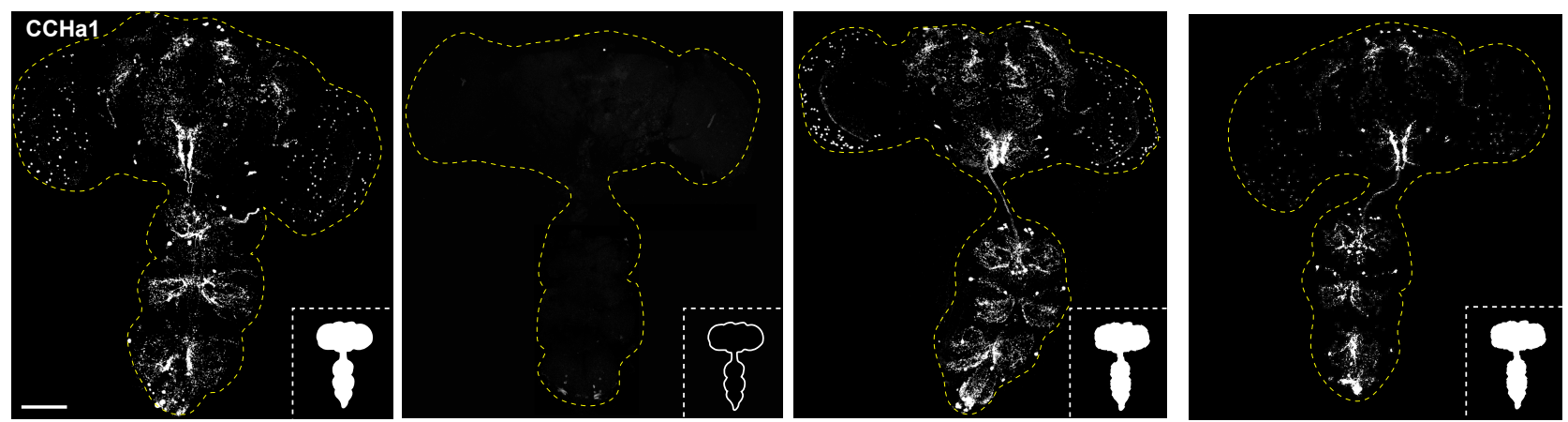

Gut
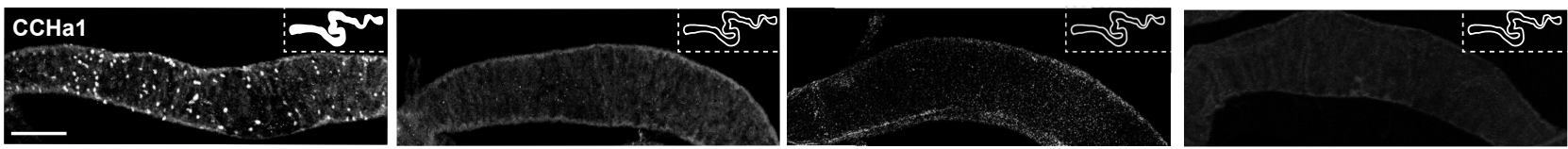

Gut-specific CCHa1 depletion

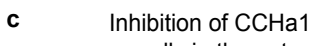

d

Activation of $\mathrm{CCHa} 1$

cells in the gut
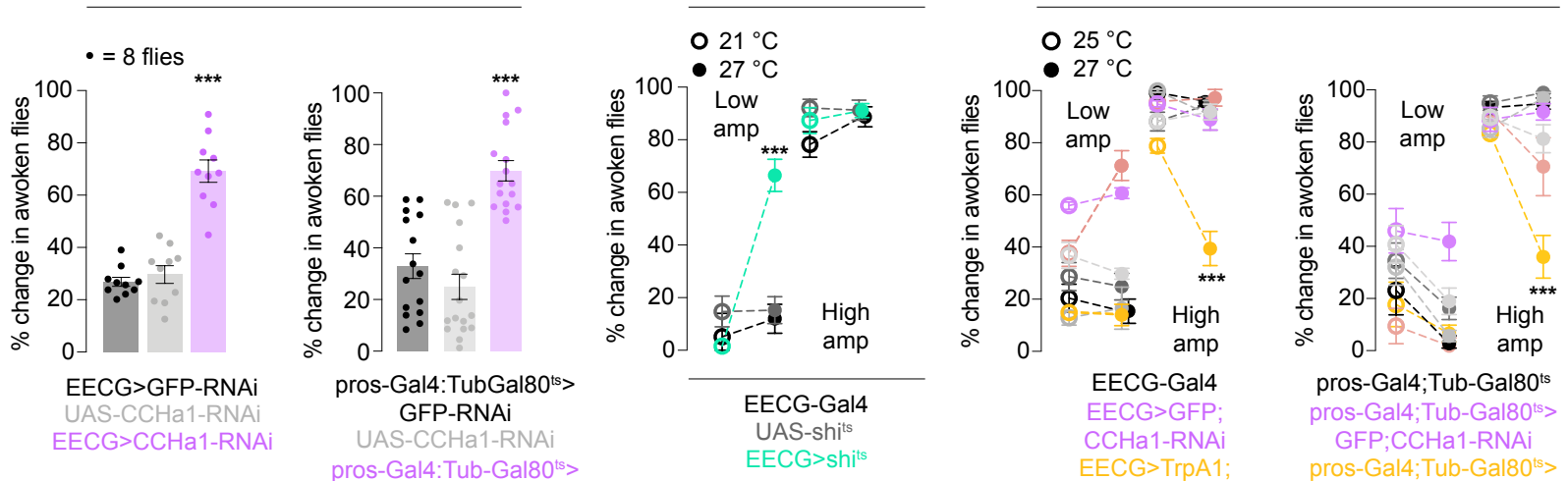

$\mathrm{CCHa} 1$

gut

EECG-Gal4

EECG $>$ GFP

CCHa1-RNAi

EECG $>$ TrpA1:

GFP-RNAi

EECG $>$ TrpA1

CCHa1-RNAi

pros-Gal4;Tub-Gal80ts pros-Gal4;Tub-Gal80ts >

GFP;CCHa1-RNAi

pros-Gal4;Tub-Gal80ts $>$

TrpA1;GFP-RNAi

pros-Gal4;Tub-Gal80ts $>$

TrpA1;CCHa1-RNAi

e

UAS-TrpA1:UAS-GFP-RNAi

UAS-TrpA1;UAS-CCHa1-RNAi 
a

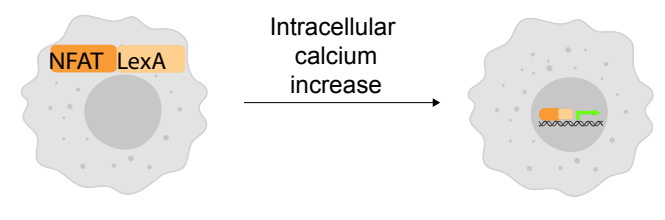

b Regular food

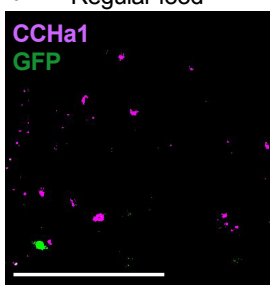

Transcriptional activation of GFP
Cell activity
$\mathrm{CCHa} 1$ protein levels
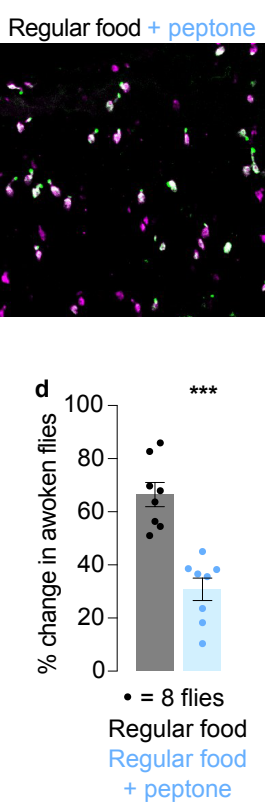

Regular food + peptone (protein)
CCHa1 mRNA levels

CCHa1 mRNA in the gut normalized to regular food

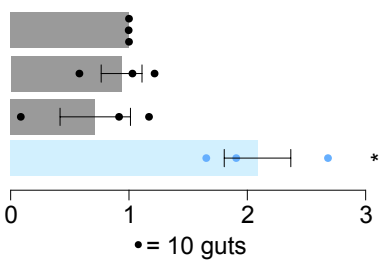

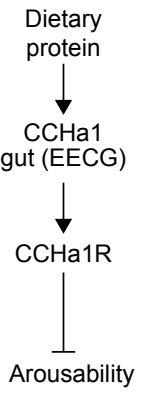

f

Regular food

Gut lumen

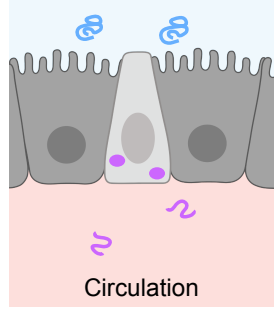

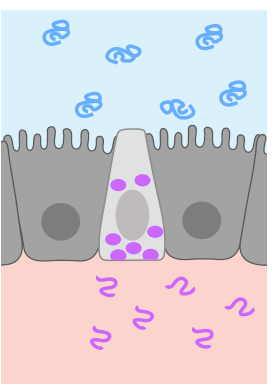

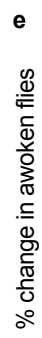

Medium amplitude $\bullet=8$ flies

Low amplitude $\quad \circ=8$ flies

Regular food

Regular food + peptone
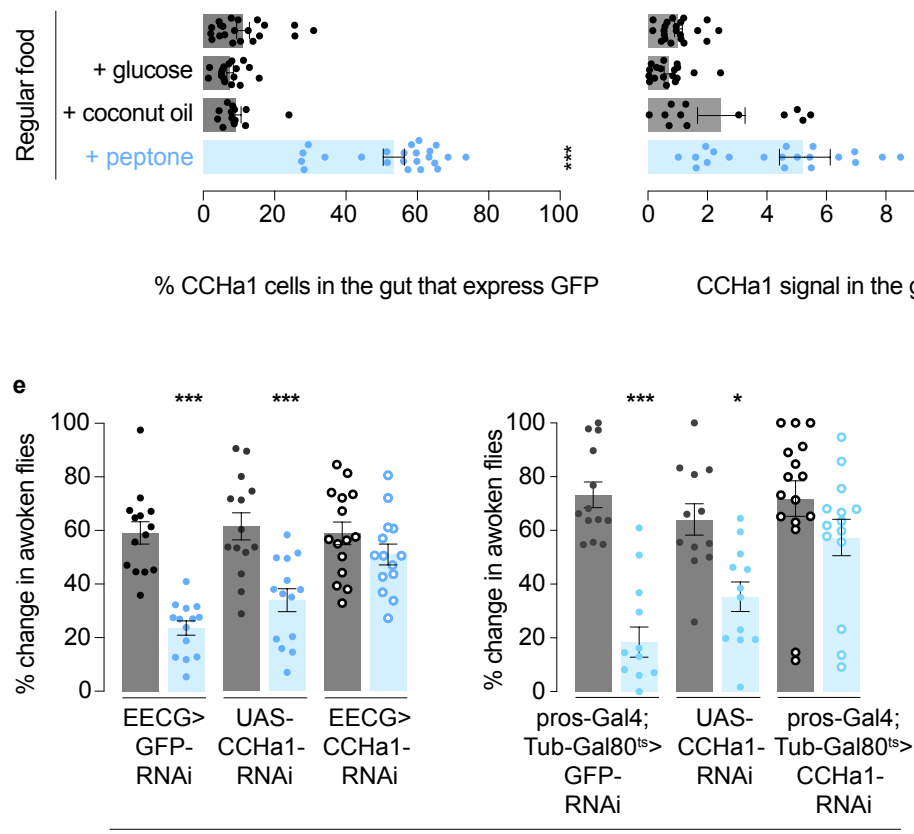
